\title{
Application of GO Management in Bistatic RCS Computation Using the Vector Parabolic Equation Method
}

\author{
X. J. Zhong, T. J. Cui, J. F. Zhang, and W. M. Yu \\ State Key Laboratory of Millimeter Waves, School of Information Science and Engineering, Southeast University, Nanjing 210096, China \\ Correspondence should be addressed to T. J. Cui; tjcui@seu.edu.cn
}

Received 2 April 2013; Revised 26 June 2013; Accepted 28 June 2013

Academic Editor: Hon Tat Hui

Copyright ( 2013 X. J. Zhong et al. This is an open access article distributed under the Creative Commons Attribution License, which permits unrestricted use, distribution, and reproduction in any medium, provided the original work is properly cited.

\begin{abstract}
The parabolic equation (PE) method is a good choice in solving large-scale problems, but the resultant matrix is usually ill conditioned. In this letter, we introduce the geometric optics (GO) management in the calculation of bistatic radar cross sections using three-dimensional vector PE method. This method manages the object surface by GO, and hence the ill-conditioned problem can be avoided. Examples are given using the presented method, original method, and the method of moments. Results show the validity and stability of the presented method.
\end{abstract}

\section{Introduction}

The parabolic equation (PE) method is extremely powerful and had been successfully used in solving long-range forward propagation problems $[1,2]$. By considering only the energy propagating along a preferred direction, the parabolic partial differential equation is used to approximate the elliptic wave equation, which can be solved by using the simple marching techniques. This method could also be used to calculate the radar cross section (RCS) of electrically large objects [3-13]. Since the paraxial direction could be chosen independently from the incident direction, the bistatic RCS of an object in a given angular sector could be computed by choosing the appropriate paraxial direction. However, the PE method usually suffers from the ill condition of the resultant matrix.

In this letter, a combination of $\mathrm{PE}$ and the geometric optics (GO) method is proposed to compute the bistatic RCS which avoids solving the ill-conditioned equations introduced by the field components coupling at the surface points. The proposed method also makes the bistatic RCS computation more robust than the original method. Examples are given to show the advantage of the proposed method.

\section{Vector Parabolic Equation Method}

In this letter, $e^{-i \omega t}$ time dependence of fields is assumed. Working with Cartesian coordinates $(x, y, z)$, the three-dimensional (3D) scalar wave equation for the scattering electric fields outside the objects could be given as

$$
\begin{aligned}
& \nabla^{2} E_{x}^{s}+k^{2} E_{x}^{s}=0, \\
& \nabla^{2} E_{y}^{s}+k^{2} E_{y}^{s}=0, \\
& \nabla^{2} E_{z}^{s}+k^{2} E_{z}^{s}=0 .
\end{aligned}
$$

Introduce the reduced functions associated with the field components:

$$
u_{m}^{s}(x, y, z)=\exp (-i k x) E_{m}^{s}(x, y, z)
$$

where $m$ may be $x, y$, or $z$. The definition of $u_{m}^{s}$ is linked to the choice of the paraxial direction $x$. Denoting $u_{m}^{s}$ by $u$, assuming the objects embedded in a vacuum, and substituting (2) into (1), $u$ could be found satisfying the following equation:

$$
\frac{\partial^{2} u}{\partial x^{2}}+2 i k \frac{\partial u}{\partial x}+\frac{\partial^{2} u}{\partial y^{2}}+\frac{\partial^{2} u}{\partial z^{2}}=0
$$

The outgoing parabolic equation for $u$ is then written as

$$
\frac{\partial u}{\partial x}=-i k(1-Q) u
$$


in which the pseudodifferential operator $Q$ is defined by

$$
Q=\sqrt{\frac{1}{k^{2}} \frac{\partial^{2}}{\partial y^{2}}+\frac{1}{k^{2}} \frac{\partial^{2}}{\partial z^{2}}+1}
$$

Using the first-order Taylor expansions of the square root and the exponential, the well-known standard parabolic equation (SPE) could be obtained:

$$
\frac{\partial u}{\partial x}=\frac{i}{2 k}\left(\frac{\partial^{2} u}{\partial y^{2}}+\frac{\partial^{2} u}{\partial z^{2}}\right) .
$$

Denote the discretization sizes along the $x, y$, and $z$ directions as $\Delta x, \Delta y, \Delta z$, and let $\Delta y=\Delta z$. To solve the partial differential equation (6), the marching technique could be used to get the solution at the range $x+\Delta x$ from the fields at range $x$. Under the assumption of homogeneous background and letting the index of refraction $n$ equal to $1,(6)$ is then discretized as

$$
\begin{array}{r}
\frac{u_{i, j}^{m+1}-u_{i, j}^{m}}{\Delta x}=\frac{i}{2 k}\left(\frac{u_{i-1, j}^{m+1}+u_{i+1, j}^{m+1}-2 u_{i, j}^{m+1}}{\Delta y^{2}}\right. \\
\left.+\frac{u_{i, j-1}^{m+1}+u_{i, j+1}^{m+1}-2 u_{i, j}^{m+1}}{\Delta z^{2}}\right) .
\end{array}
$$

The above formula could also be given as below

$$
a u_{i-1, j}^{m+1}+b u_{i, j-1}^{m+1}+c u_{i, j}^{m+1}+d u_{i, j+1}^{m+1}+e u_{i+1, j}^{m+1}=u_{i, j}^{m}
$$

in which

$$
\begin{gathered}
a=b=d=e=-\frac{i \Delta x}{2 k \Delta z^{2}}, \\
c=1+\frac{i 2 \Delta x}{\Delta y^{2} k}
\end{gathered}
$$

Using the iterative solver such as BICGSTAB to solve the equations formed by (8), the fields at range $x+\Delta x$ could be obtained from the fields at range $x$.

\section{GO Management at Surface Points}

In the vector parabolic equation (VPE) method, to consider the field components coupling on the object surfaces, it needs to solve equations at the object surface points [3]:

$$
\begin{gathered}
n_{x} E_{y}(P)-n_{y} E_{z}(P)=0, \\
n_{x} E_{z}(P)-n_{z} E_{x}(P)=0, \\
n_{y} E_{x}(P)-n_{x} E_{y}(P)=0, \\
\nabla \cdot \vec{E}(P)^{s}=0,
\end{gathered}
$$

where $E_{x}, E_{y}, E_{z}$ are the electric field components at the surface point $P, \vec{E}(P)$ is the total electric field at $P$, and $\widehat{n}=$ $\widehat{x} n_{x}+\widehat{y} n_{y}+\widehat{z} n_{z}$ is the outward unit normal vector at $P$.
Now, the GO management is introduced to avoid solving the above equations. Let $\hat{i}$ be the incident unit vector and $\hat{r}$ be the reflection unit vector. Then the total incident electric field could be decomposed as

$$
\begin{aligned}
& E_{i}^{\mathrm{TE}}(P)=\vec{E}(P) \cdot \widehat{\phi}_{i}^{\mathrm{TE}} \\
& E_{i}^{\mathrm{TM}}(P)=\vec{E}(P) \cdot \widehat{\phi}_{i}^{\mathrm{TM}}
\end{aligned}
$$

in which

$$
\begin{gathered}
\widehat{\phi}_{i}^{\mathrm{TE}}=\frac{\widehat{n} \times \hat{i}}{|\widehat{n} \times \hat{i}|}, \\
\widehat{\phi}_{i}^{\mathrm{TM}}=\frac{\widehat{\phi}_{i}^{\mathrm{TE}} \times \widehat{i}}{\left|\widehat{\phi}_{i}^{\mathrm{TE}} \times \hat{i}\right|} .
\end{gathered}
$$

Let $\mathrm{Rv}$ and $\mathrm{Rh}$ be the reflection coefficients of the TE and TM fields on the object surface, respectively, and DF be the divergence factor at the point. The reflected TE and TM fields could be obtained by

$$
\begin{aligned}
& E_{r}^{\mathrm{TE}}(P)=E_{i}^{\mathrm{TE}}(P) \cdot \mathrm{Rv} \cdot \mathrm{DF}, \\
& E_{r}^{\mathrm{TM}}(P)=E_{i}^{\mathrm{TM}}(P) \cdot \mathrm{Rh} \cdot \mathrm{DF} .
\end{aligned}
$$

Furthermore, the total reflected field could be given as

$$
\vec{E}_{r}(P)=E_{r}^{\mathrm{TE}}(P) \cdot \widehat{\phi}_{r}^{\mathrm{TE}}+E_{r}^{\mathrm{TM}}(P) \cdot \widehat{\phi}_{r}^{\mathrm{TM}},
$$

where

$$
\begin{gathered}
\widehat{\phi}_{r}^{\mathrm{TE}}=\frac{\widehat{n} \times \widehat{r}}{|\widehat{n} \times \widehat{r}|}, \\
\widehat{\phi}_{r}^{\mathrm{TM}}=\frac{\widehat{\phi}_{r}^{\mathrm{TE}} \times \widehat{r}}{\left|\widehat{\phi}_{r}^{\mathrm{TE}} \times \widehat{r}\right|} .
\end{gathered}
$$

So the major steps of using GO management are as follows: firstly, acquire the incident field on the object surface according to the incident angle; secondly, decompose the incident field using formula (11); thirdly, calculate the total reflected field using formulas (13) and (14).

Once the total reflected field is obtained, the initial scattering field at each direction is determined.

\section{RCS Calculations}

The RCS calculation could be processed using the marching techniques with formula (8). At each range $x$ where the objects are involved, the GO management should be taken after the marching process is finished so that the field components coupling effects on the object surfaces could be considered. Since the marching technique is used, the computation complexity of the proposed method is mainly in proportion to the transverse size of the objects. Hence the method could solve the scattering problems of very large objects on single personal computers.

Examples are given to verify the presented method. The first example is a perfectly electrical conducting (PEC) cuboid 


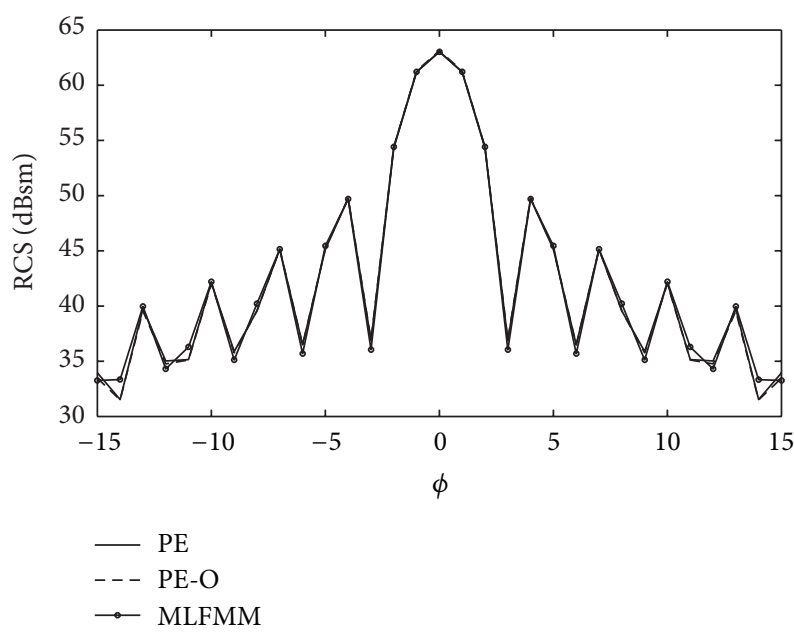

Figure 1: RCS of a PEC cuboid calculated by PEs and MLFMA.

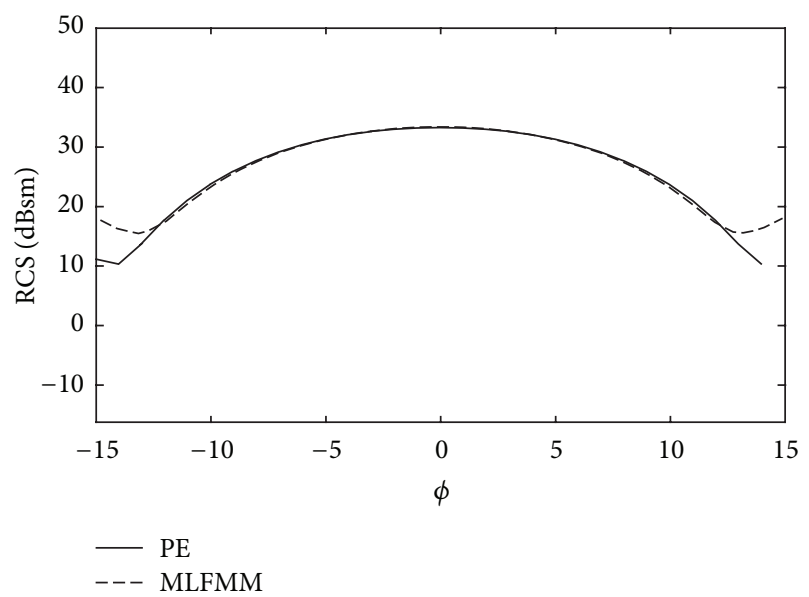

Figure 2: RCS of a PEC cylinder calculated by PE and MLFMA.

with thickness of $0.1 \mathrm{~m}$, height of $20 \mathrm{~m}$, and length of $20 \mathrm{~m}$. The thickness direction is along $x$. The incident signal is $\vec{E}=$ $e^{i \vec{k} \cdot \vec{z}} \widehat{z}$ with the incident angle of $(\pi / 2,0), \vec{k}=-\widehat{x}$, operating at the frequency of $300 \mathrm{MHz}$.

Figure 1 shows the RCS calculated by PE methods, in which PE denotes the result of PE with GO management, PE$\mathrm{O}$ denotes the original PE method [3], and $\phi$ is the receiving angle with the unit of degree. As comparison, the full-wave method of moments is also used to calculate the problem, in which the multilevel fast multipole method (MLFMM) $[14,15]$ has been adopted to accelerate calculations. The CPU (Xeon E5420@2.5 GHz) times used by PE methods are both about ten seconds (the iteration number of bicgstab codes for $\mathrm{PE}$ is 6 and for PE-O is 8 ; the relative residual is $1 e-6$ ), while the CPU time for MLFMM is about 4180 seconds. Though much fast as they are, the results calculated by the PE methods have excellent agreements to those from MLFMM, especially in the paraxial direction.

A PEC cylinder with the radius of $2.0 \mathrm{~m}$, height of $3.0 \mathrm{~m}$, and the axis along the $z$ direction is also calculated using the same incident signal. Figure 2 gives the results from PE

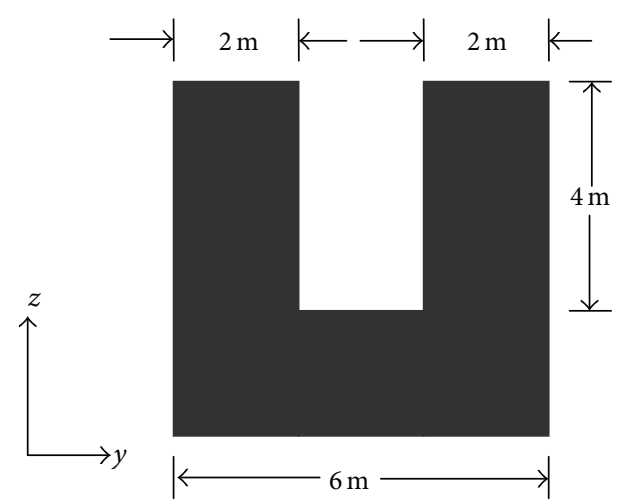

Figure 3: The cross section of a PEC object.

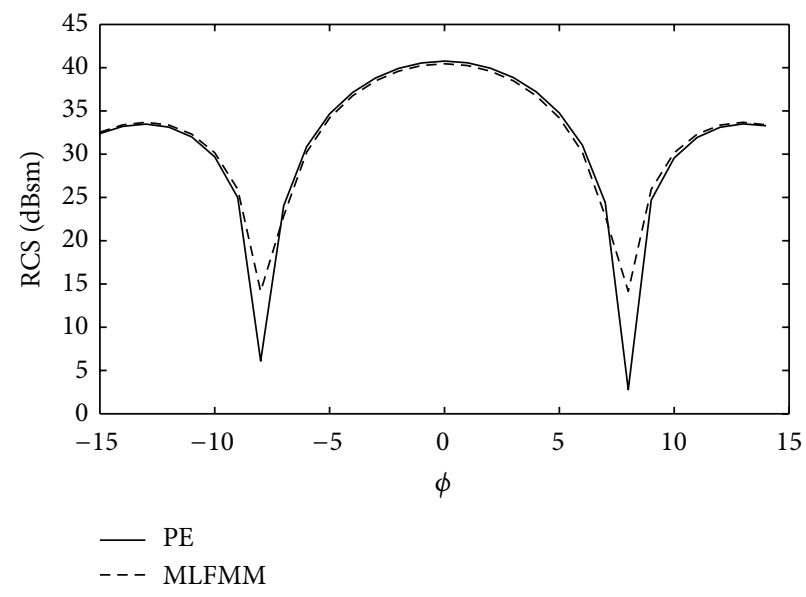

FIgURE 4: RCS of the PEC object shown in Figure 3 calculated from PE and MLFMM.

and MLFMM. Different from the first example, the original PE method failed to complete the computation due to bad condition numbers. From Figure 2, it is shown that the RCS calculated by PE (with iteration number of 25 , the relative residual is $1 e-6$ ) coincides very well with that from MLFMM. The difference between PE and MLFMM results is little larger on the directions deviate from the paraxial direction; this is caused by the shortcoming of the PE method, and the larger angle deviated from the paraxial direction, the less accurate the results would be. This problem could be alleviated through changing the paraxial direction. We also observe that the PE method could be more accurate when the objects become larger, and an example of electrical large object is shown later.

To further validate the proposed method, a complicated PEC object with the cross section was shown in Figure 3 and the thickness of $0.3 \mathrm{~m}$ is also considered under the same incident signal. Figure 4 illustrates the results from PE and MLFMM, which have also excellent agreements.

Finally, a PEC square plate with the length of $100 \mathrm{~m}$ is considered to test the computation capability when an electrically large object is involved, as shown in Figure 5. Under the illumination of the same incident field, the computed RCS by VPE and MLFMM are illustrated in Figure 6, from which 


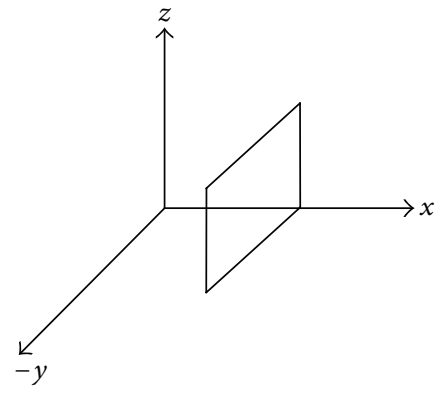

Figure 5: PEC square plate with length of $100 \mathrm{~m}$.

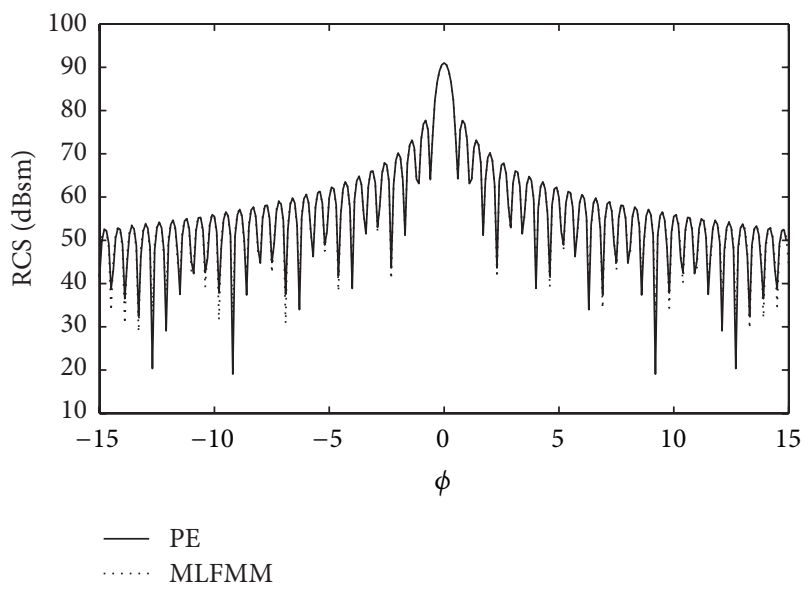

FIgURE 6: RCS of PEC square calculated by PE and MLFMM.

we observe that the computational results have excellent agreements. Although the object is electrically large, it takes less than two minutes to compute the results using the VPE method on a PC.

\section{Conclusion}

The RCS computation is accelerated by using the VPE formulas together with the GO management at the object surfaces. Since it does not need to solve equations involved at the objects' surfaces, the computation is very efficient and robust and could handle more complex objects than original PE method. Numerical examples show the validity and capability of the proposed method.

\section{References}

[1] G. D. Dockery and J. R. Kuttler, "An improved impedanceboundary algorithm for fourier split-step solutions of the parabolic wave equation," IEEE Transactions on Antennas and Propagation, vol. 44, no. 12, pp. 1592-1599, 1996.

[2] J. R. Kuttler and G. D. Dockery, "Theoretical description of the parabolic approximation/Fourier split-step method of representing electromagnetic propagation in the troposphere," Radio Science, vol. 26, no. 2, pp. 381-393, 1991.

[3] A. A. Zaporozhets and M. F. Levy, "Bistatic RCS calculations with the vector parabolic equation method," IEEE Transactions on Antennas and Propagation, vol. 47, no. 11, pp. 1688-1696, 1999.

[4] M. F. Levy and P.-P. Borsboom, "Radar cross-section computations using the parabolic equation method," Electronics Letters, vol. 32, no. 13, pp. 1234-1236, 1996.

[5] M. F. Levy, P. P. Borsboom, A. A. Zaporozhets, and A. Z. Hyaric, "RCS calculations with the parabolic wave equation," in Proceedings of the AGARD SPP Symposium on Radar Signature Analysis and Imaging of Military Targets, no. 583, pp. 5.1-5.9, Ankara, Turkey, October 1996.

[6] M. F. Levy and A. A. Zaporozhets, "Target scattering calculations with the parabolic equation method," Journal of the Acoustical Society of America, vol. 103, no. 2, pp. 735-741, 1998.

[7] A. R. Mallahzadeh, M. Soleimani, and J. Rashed-Mohassel, "RCS computation of airplane using parabolic equation," in Proceedings of the Asia-Pacific Microwave Conference (APMC '05), December 2005.

[8] J. P. Bérenger, "A perfectly matched layer for the absorption of electromagnetic waves," Journal of Computational Physics, vol. 114, no. 2, pp. 735-741, 1994.

[9] A. A. Zaporozhets and M. F. Levy, "Radar cross-section calculation with marching methods," Electronics Letters, vol. 34, no. 20, pp. 1971-1972, 1998.

[10] Z. X. Huang, Q. Wu, and X. L. Wu, "Solving multi-object radar cross section based on wide-angle parabolic equation method," Journal of Systems Engineering and Electronics, vol. 17, no. 4, pp. 722-724, 2006.

[11] H. T. G. Woo, M. J. Schuh, and M. L. Sanders, "Benchmark radar targets for the validation of computational electromagnetics programs," IEEE Antennas and Propagation Magazine, vol. 35, no. 1, pp. 84-89, 1993.

[12] A. A. Zaporozhets A, "Current marching technique for electromagnetic scattering computations," IEEE Transactions on Antennas and Propagation, vol. 47, no. 6, pp. 1016-1024, 1999.

[13] M. D. Collins and M. F. Werby, "A parabolic equation model for scattering in the ocean," Journal of the Acoustical Society of America, vol. 85, no. 5, pp. 1895-1902, 1989.

[14] N. Engheta, W. D. Murphy, V. Rokhlin, and M. S. Vassiliou, "The fast multipole method (FMM) for electromagnetic scattering problems," IEEE Transactions on Antennas and Propagation, vol. 40, no. 6, pp. 634-641, 1992.

[15] J. Song, "Multilevel fast multipole algorithm for electromagnetic scattering by large complex objects," IEEE Transactions on Antennas and Propagation, vol. 45, no. 10, pp. 1488-1493, 1997. 

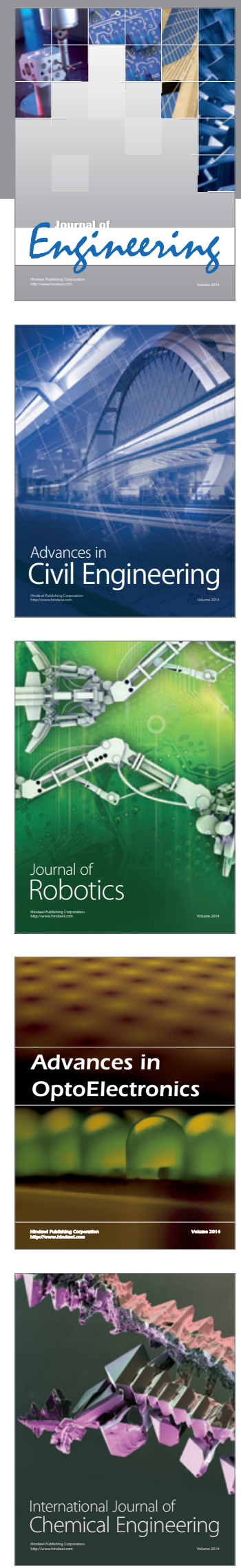

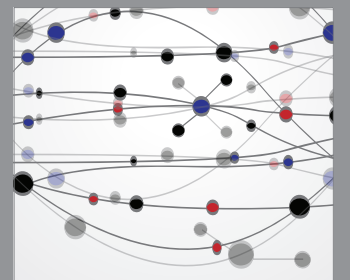

The Scientific World Journal
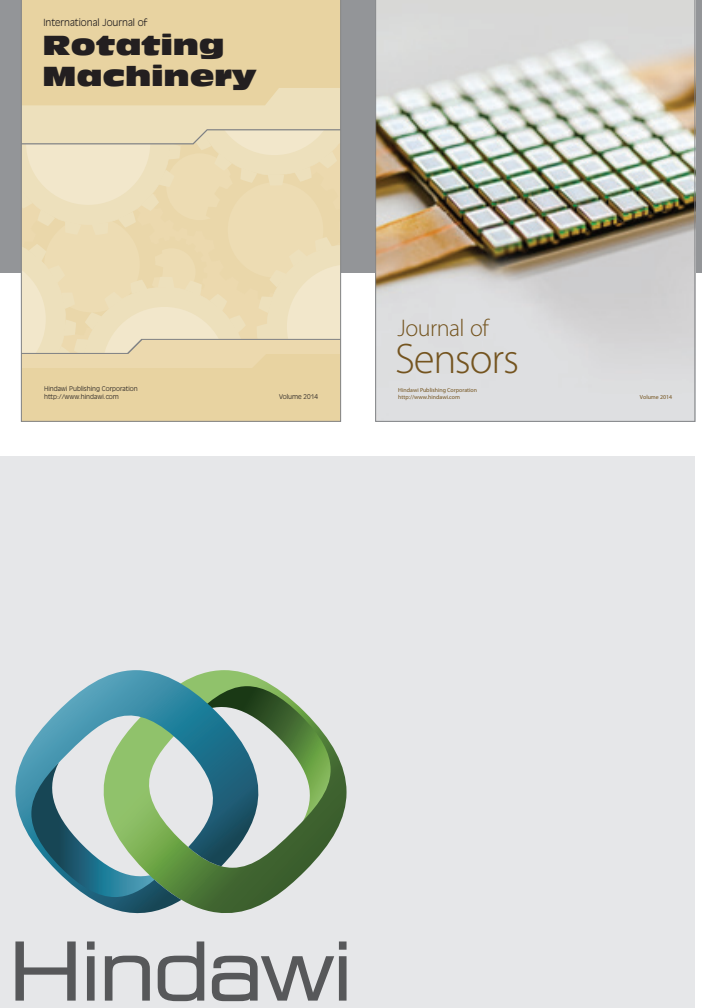

Submit your manuscripts at http://www.hindawi.com
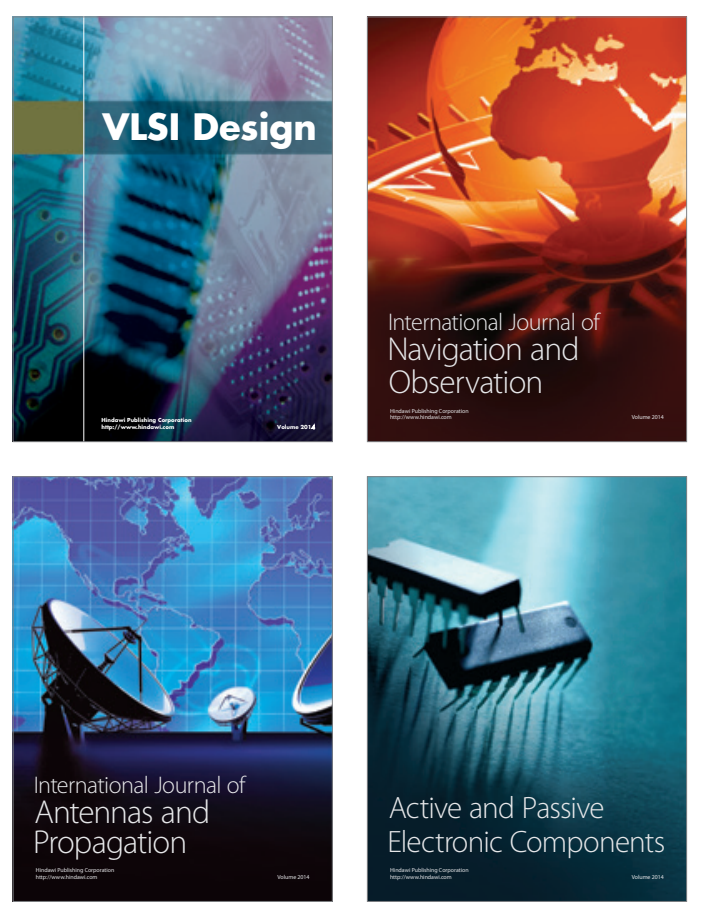
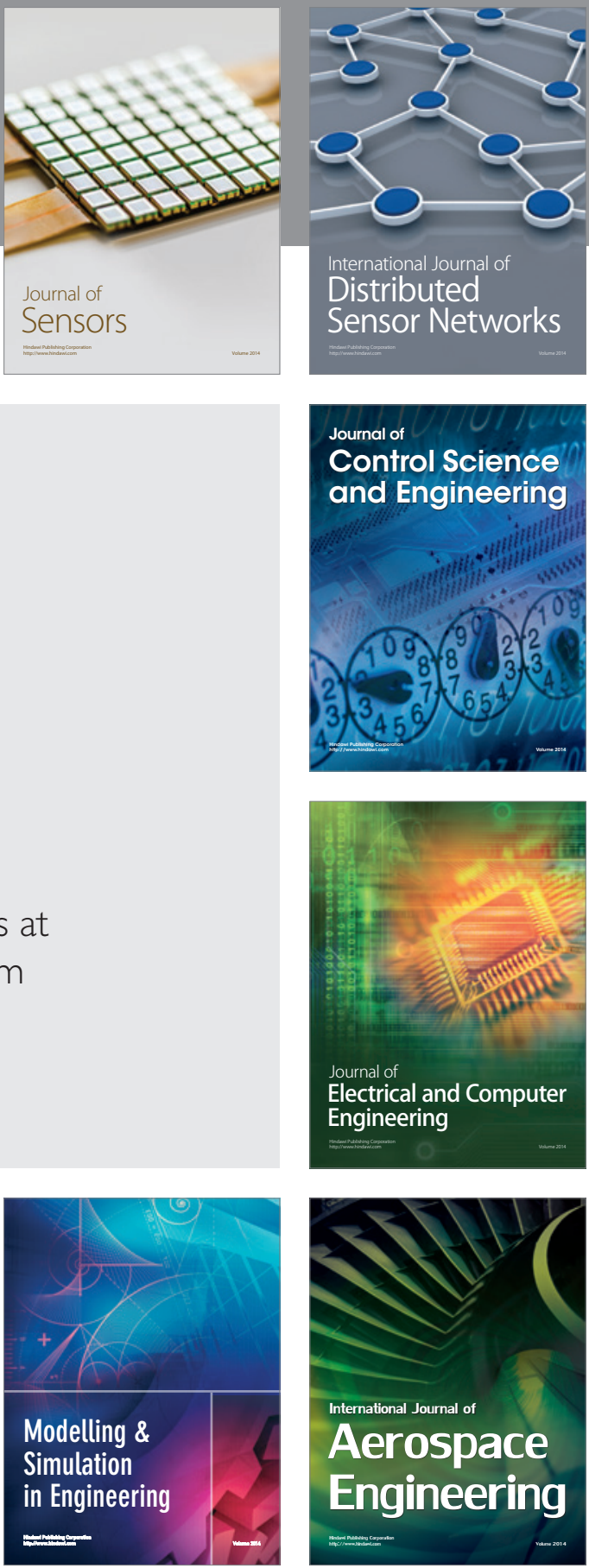

Journal of

Control Science

and Engineering
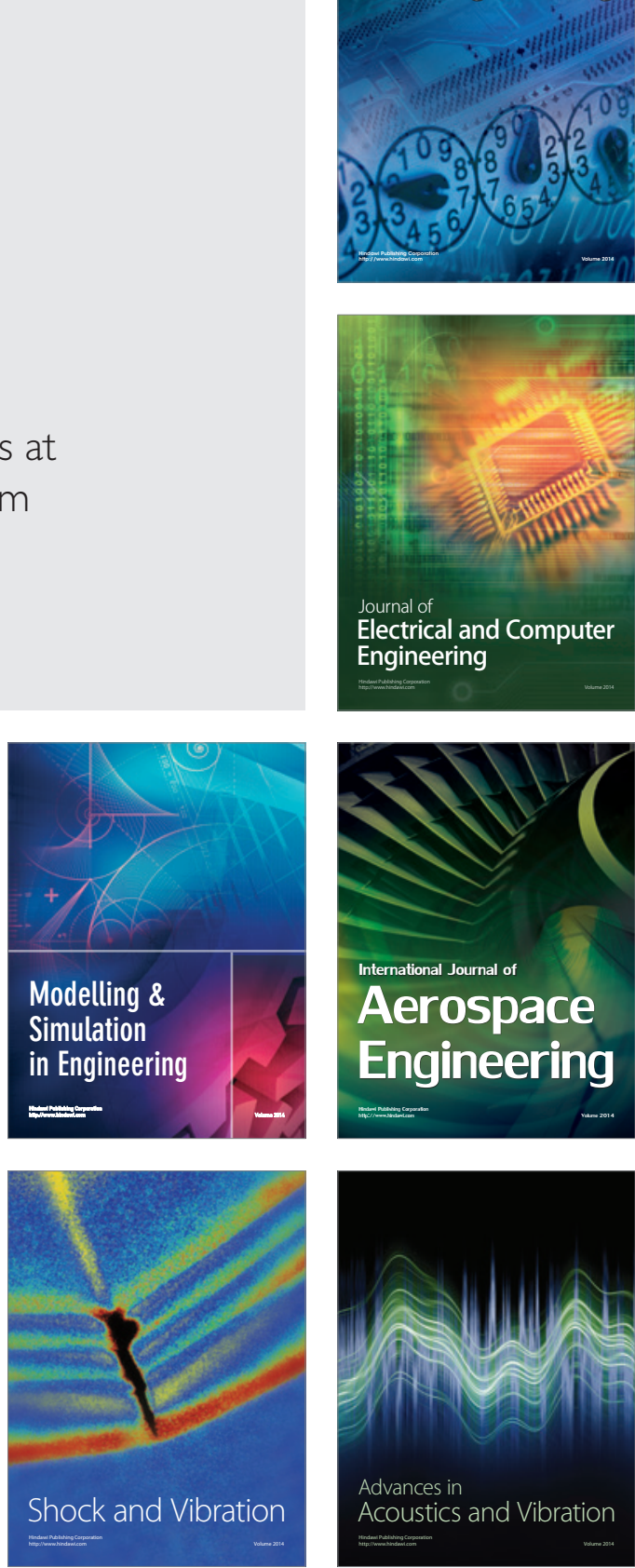